\title{
Far Ultraviolet Spectroscopic Explorer observations of high-velocity gas associated with the Monoceros Loop SNR
}

\author{
B. Y. Welsh ${ }^{1}$, D. M. Sfeir ${ }^{1}$, S. Sallmen ${ }^{1}$, and R. Lallement ${ }^{2}$ \\ 1 Experimental Astrophysics Group, Space Sciences Laboratory, UC Berkeley, Berkeley, CA 94720, USA \\ 2 Service d'Aéronomie du CNRS, 91371 Verrières-le-Buisson, France
}

Received 14 February 2001 / Accepted 2 April 2001

\begin{abstract}
We present Far Ultraviolet Spectroscopic Explorer (FUSE) observations of high-velocity gas $\left(V_{\mathrm{LSR}}=\right.$ $+65 \mathrm{~km} \mathrm{~s}^{-1}$ ) seen towards the star HD 47240 which lies just behind the Monoceros Loop Supernova Remnant at a distance of $\sim 1800 \mathrm{pc}$. This high-velocity absorption feature is detected in the far ultraviolet lines of O I, Ar I, N I, C I, Fe II and P II, in addition to being detected at visible wavelengths in Na I and Ca II and at near ultraviolet wavelengths in $\mathrm{Mg}$ II, Mg I, S II, O I, Si II, C II*, Al II and Fe II. High-velocity interstellar gas has not been detected in the high-ionization (high-temperature) species of O VI, C IV and Si IV. Gas phase abundances relative to that of sulphur for this high velocity feature have been derived. The refractory elements of $\mathrm{Fe}, \mathrm{Si}$ and $\mathrm{Al}$ are all less depleted than that normally found for cold disk gas in the interstellar medium, with a pattern of relative abundance more similar to that of warm interstellar disk gas. However, the elements of N, $\mathrm{O}$, and Ar show an opposite pattern of relative depletion in which their apparent elemental deficiency may be attributed to ionization effects, as also found for high-velocity gas associated with the Vela SNR by Jenkins et al. (1998). The lack of detection of high-ionization gas at high velocity suggests that the Monoceros Loop remnant is more evolved than other remnants such as the Vela SNR or Cygnus Loop, and that an age of 30 000-150 000 years seems appropriate.
\end{abstract}

Key words. ISM: supernova remnants - ISM: abundances

\section{Introduction}

The NASA Far Ultraviolet Spectroscopic Explorer (FUSE) was launched on June 24th 1999, and its instrumentation is ideally suited to probe interstellar plasma existing in a wide range of different physical conditions using the many astrophysically important absorption lines present throughout the 912 to $1187 \AA$ wavelength region (Moos et al. 2000). A particularly interesting region for FUSE to study highly disturbed interstellar gas is the Monoceros Loop Supernova Remnant (SNR). This is a well evolved remnant of age $\sim 10^{5}$ years, is some $100 \mathrm{pc}$ in diameter and lies at a distance of $\sim 1.6 \mathrm{kpc}$ (Odegard 1986). The study of SNRs is important for understanding both the energy and ionization balance of the interstellar medium, since supernova explosions and strong stellar winds from OB stars are the dominant sources of hightemperature and high-velocity shocked gas in the interstellar medium (ISM). Much of the energy released in a supernova explosion is deposited in the kinetic energy of the ejecta which subsequently interacts with the ambient interstellar gas.

Send offprint requests to: B. Y. Welsh, e-mail: bwelsh@ssl. berkeley.edu
The emission characteristics of the gas associated with the Monoceros Loop SNR have been well studied at radio (Graham et al. 1982), X-ray (Leahy et al. 1986), $\gamma$ ray (Jaffe et al. 1997) and visible (Fesen et al. 1985) wavelengths. These and other studies indicate that the Monoceros Loop is probably interacting with the adjacent (and nearer) Rosette Nebula (NGC 2244), which contains a cluster of several ionizing early O-type stars (NGC 2264) known to be losing appreciable amounts of mass to the ambient ISM (Kuchar \& Bania 1993). Remarkably for this well-known region, only one study of the Monoceros Loop/Rosette Nebula complex has been undertaken using interstellar absorption lines. Wallerstein \& Jacobsen (1976) (hereafter WJ) have observed 25 stars in the direction of this SNR region using measurements of the interstellar Ca II K-line (3393 A) and Na I D-lines (5890 $\AA)$ taken at a spectral resolution of $12 \mathrm{~km} \mathrm{~s}^{-1}$. These observations revealed high-velocity absorption features at $V_{\mathrm{LSR}}=+69 \mathrm{~km} \mathrm{~s}^{-1}$ towards the star HD 47240 and another feature at $V_{\mathrm{LSR}}=-31 \mathrm{~km} \mathrm{~s}^{-1}$ towards HD 47359 , both of which have been associated with the expansion of the nebular gas.

Although the gross kinematical properties of the Monoceros Loop/Rosette Nebula region are well documented, the detailed interactions of the associated 
complex ionized, neutral and molecular gas structures interaction are still much debated. For example, although WJ detected gas cloud components with 5 different velocity values towards the 25 stars that they observed, only two of the velocities of these components were common to those found by H I $21 \mathrm{~cm}$ observations. Furthermore, both of the high-velocity cloud components observed by WJ in the Na I and Ca II lines towards HD 47240 and HD 47359 have anomalous Na I/Ca II ratios < 1.0, which is consistent with an enhancement of interstellar Ca due to the destruction of ambient interstellar dust grains by the passage of high-velocity shocks (Siluk \& Silk 1976). Observations of stars associated with the Vela SNR by Danks \& Sembach (1995) have shown similarly low values of the Na I/Ca II ratio for interstellar gas clouds with velocities up to $\sim 60 \mathrm{~km} \mathrm{~s}^{-1}$. UV observations of the same remnant have revealed that the gas at high velocity exhibits higher than normal ionization and has unusually low abundances of N I and O I (Jenkins et al. 1998), whereas the depletion of $\mathrm{Al}, \mathrm{Si}$ and $\mathrm{Fe}$ in high velocity interstellar gas clouds is substantially less (Jenkins et al. 1984).

As part of a long-term program using the FUSE satellite in which we hope to observe the absorption characteristics of the disturbed interstellar medium towards 4 earlytype stars in the direction of Monoceros Loop SNR, we currently present preliminary observations of high velocity interstellar gas absorption seen towards the B1Ib star HD $47240\left(M_{v}=6.2, E(B-V)=0.31\right)$ which lies just behind the south central region of the SNR at a distance of $\sim 1800$ pc. These far ultraviolet data have been supplemented with archival near ultraviolet and newly presented high resolution visible absorption line data taken towards this star to further understand the physical and chemical nature of the high-velocity interstellar gas clouds associated with this expanding supernova remnant.

\section{Observations and data processing}

The spectral integrations accumulated with the FUSE instrument were obtained in the histogram integration mode on March 15th, 2000. The observations were performed with the star centered in the $30 \times 30$ arcsec aperture of the LiF1 channel of the FUSE spectrograph, but due to observational difficulties no data were obtained in the FUSE $\mathrm{SiC}$ spectral channels (which cover the short wavelength end of the bandpass). Thus, the far UV data presented in this paper consists of eleven separate, $500 \mathrm{~s}$ long integrations that cover the $990-1187 \AA$ wavelength region, and all data have been individually processed using version 1.8.3 of the FUSE science data reduction (CALFUSE) pipeline. This processing of the detector raw histogram data nominally accounts for geometric image distortions, background level subtraction, image drift due to instrument thermal problems, detector deadtime, wavelength and flux calibration (Sahnow et al. 2000).

Each of the 11 processed spectra were co-added and averaged using standard IDL data reduction routines. The data contained in the LiF1a and LiF1b detector seg- ments were deemed to be of the best quality and these were subsequently used in the following spectral analysis. (However, we note that the LiF2a and LiF2b spectra were used to check for consistency in all the line detections that we shall now report.) For the purposes of this preliminary paper we have restricted our analysis to the two wavelength regions shown in Figs. 1 and 2 that cover the 1030-1050 A and 1130-1155 $\AA$ ranges respectively.

The zero point of the wavelength scale was determined with reference to the many $\mathrm{H}_{2}$ molecular lines that were detected in both spectral regions. We have assumed that these lines occur at the same velocity as that of the visible $\mathrm{CH}$ molecular line at $4232.5 \AA$ (i.e. $V=+3 \mathrm{~km} \mathrm{~s}^{-1}$ ), as observed at a spectral resolution of $5 \mathrm{~km} \mathrm{~s}^{-1}$ by Sfeir (1999). For the convenience of comparison with radio data, all velocities in this paper are in the local standard of rest (LSR) frame. The resultant FUSE spectra typically have a $S / N$ ratio $>15: 1$ and a velocity resolution of $\sim 13 \mathrm{~km} \mathrm{~s}^{-1}$ as determined from absorption line-profile fitting of the weak interstellar lines of $\mathrm{H}_{2}$ at 1041.2 $\AA$ and 1047.6 $\AA$.

In addition, we have supplemented the FUSE far UV data with high spectral resolution observations of the $\mathrm{Na}$ I

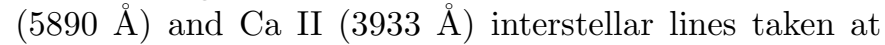
the Lick Observatory and with ultraviolet observations of HD 47240 extracted from the on-line IUE data archive (Rodriguez-Pascual et al. 1999) that have a spectral resolution of $\sim 15 \mathrm{kms}^{-1}$, which is comparable to that of the FUSE data. Further details of the visible and IUE data gained towards HD 47240 and other stars in the Monoceros Loop SNR have been presented in the Ph.D. Thesis of Sfeir (1999).

\section{Interstellar analysis}

Inspection of the FUSE absorption spectra shown in Figs. 1 and 2 reveal significant detections of the important interstellar absorption line of O VI (1031.9 $\AA)$, as well as detections of the ground state resonance lines of interstellar O I (1039.2 ̊), C I (1139.8 ̊), Ar I (1048.2 ̊), N I (1134.9 ̊), Fe II (1143.23\& $1144.9 \AA)$ and P II (1152.8 $\AA$ ). It is immediately apparent from these line profiles that many of the strongest absorption lines are accompanied by a well resolved high-velocity (HV) component at $V_{\mathrm{LSR}} \sim+65 \mathrm{~km} \mathrm{~s}^{-1}$. We identify this as the high velocity component observed by WJ in both the Na I and Ca II interstellar lines at $V_{\mathrm{LSR}}=+69 \mathrm{kms}^{-1}$ towards HD 47240.

Unfortunately we do not uniquely detect the $V_{\mathrm{LSR}}=$ $-31 \mathrm{~km} \mathrm{~s}^{-1}$ component (that was seen in the visible $\mathrm{Na} \mathrm{I}$ lines towards HD 47359 by WJ and in Ca II towards HD 47240 by Sfeir 1999) in our present FUSE spectra. This is presumably due to blending of this component with the strong central line-of-sight absorption at $V_{\mathrm{LSR}}=$ $+3 \mathrm{~km} \mathrm{~s}^{-1}$ that is not resolved by the FUSE instrument. However, we note that the asymmetry in the blue-wing of the weak Fe II $1143.23 \AA$ line is most probably due to an absorption component at $\sim-30 \mathrm{~km} \mathrm{~s}^{-1}$. Additionally, we also detect many of the lines associated with the 


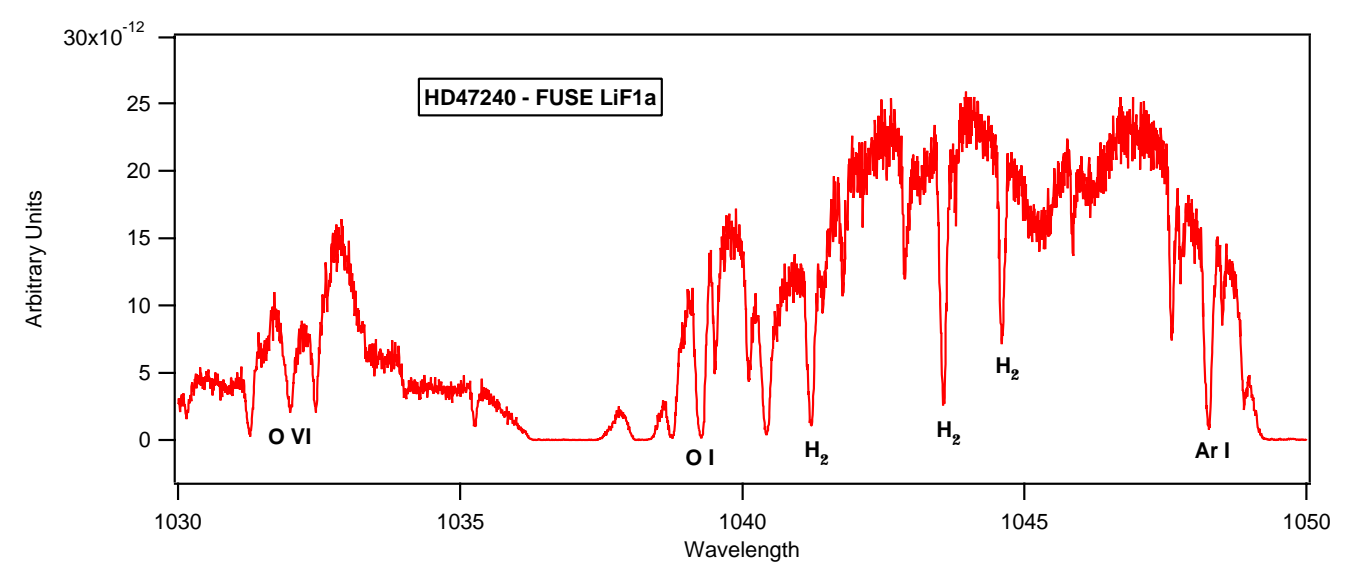

Fig. 1. HD 47240 - Average FUSE LiF1a wavelength corrected spectrum.

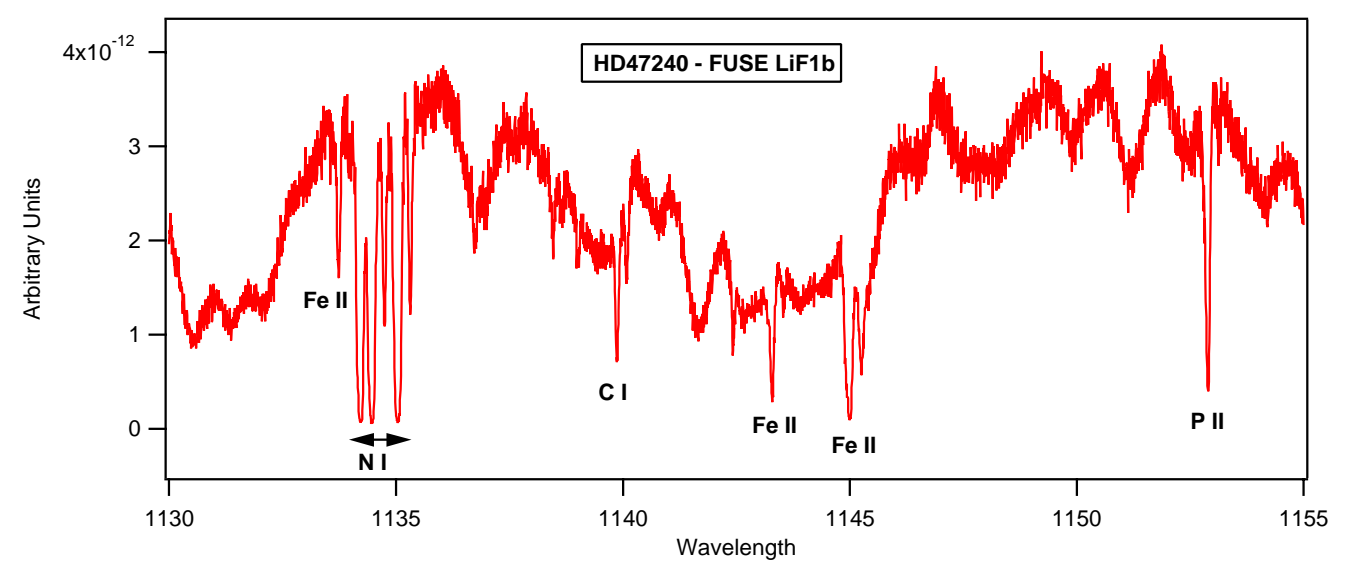

Fig. 2. HD 47240 - Average FUSE LiF1b wavelength corrected spectrum.

Lyman (B-X) and Werner (C-X) bands of the $\mathrm{H}_{2}$ molecule in these FUSE spectra (some of which are also accompanied by an $\mathrm{HV}$ component at $+65 \mathrm{~km} \mathrm{~s}^{-1}$ ) whose detailed analysis will be deferred to a future paper.

For each of the atomic lines that are accompanied by a detection of the $\mathrm{HV}$ component we have fitted their local stellar continua with a multi-order polynomial to produce a residual intensity profile. The placement and shape of a stellar continuum was guided by those of the many stellar absorption lines observed throughout the FUSE spectrum. The interstellar absorption profiles were then fit with one or more absorption components (i.e. "clouds") using line oscillator strengths listed by Morton (2001). This fitting procedure is discussed in detail by Sfeir et al. (1999), such that each theoretical absorption profile is described by a gaussian velocity dispersion parameter, $b$, a cloud component LSR velocity, $V$, and a cloud column density, $N$. These best-fit values of $V, b$ and $N$ for all the detected interstellar lines are also listed (together with their respective errors and equivalent width values) in Table 1.

In Fig. 3 we show the FUV residual intensity profiles that possess an $\mathrm{HV}$ feature together with their respective best-fit profile models. Note that since the strong, central components of these line profiles (which occur at $V_{\mathrm{LSR}} \sim 3 \mathrm{kms}^{-1}$ ) are all saturated and their best-fit col- umn densities are thus highly uncertain, we present only the fit data to the well resolved, weaker high velocity component for each line species.

In Figs. 4 and 5 we also show the high resolution absorption profiles of the $\mathrm{Na}$ I and $\mathrm{Ca}$ II lines towards HD 47240 (from Sfeir 1999) and in Fig. 6 we show the near ultraviolet absorption profiles of the Al II (1670 $)$ ), Si II $(1304 \AA)$, S II $(1253 \AA)$, Fe II $(1608 \AA)$, C II* $(1336 \AA), \operatorname{Mg}$ I $(2852 \AA)$ and $\mathrm{Mg}$ II (2800 $\AA$ doublet) lines as recorded by $I U E$.

We note that the positive high velocity component seen in the FUSE data is also detected at a very similar velocity in many of the profiles of the strongest absorption lines recorded by the IUE satellite and also in the ground based data. The best fit values for $V, b$ and $N$ for the (partially resolved) $\mathrm{HV}$ components of these lines are listed in Tables 2 and 3, and their derivations have been discussed in detail in Sfeir (1999). We note that the column density values derived from both the IUE and FUSE spectral lines for $N$ (Fe II) are (within the errors) in general agreement. Since the FUSE derivation was obtained using a constrained fit to the Fe II doublet at $1144 \AA$ we believe this to be a more accurate determination of column density and thus we use $N(\mathrm{Fe} \mathrm{II})=13.75 \pm 0.08 \mathrm{~cm}^{-2}$ in all future calculations. For the case of $N(\mathrm{OI})$, we use the valued derived from the FUV line of $1039.2 \AA$ since the OI $1302 \AA$ line is 

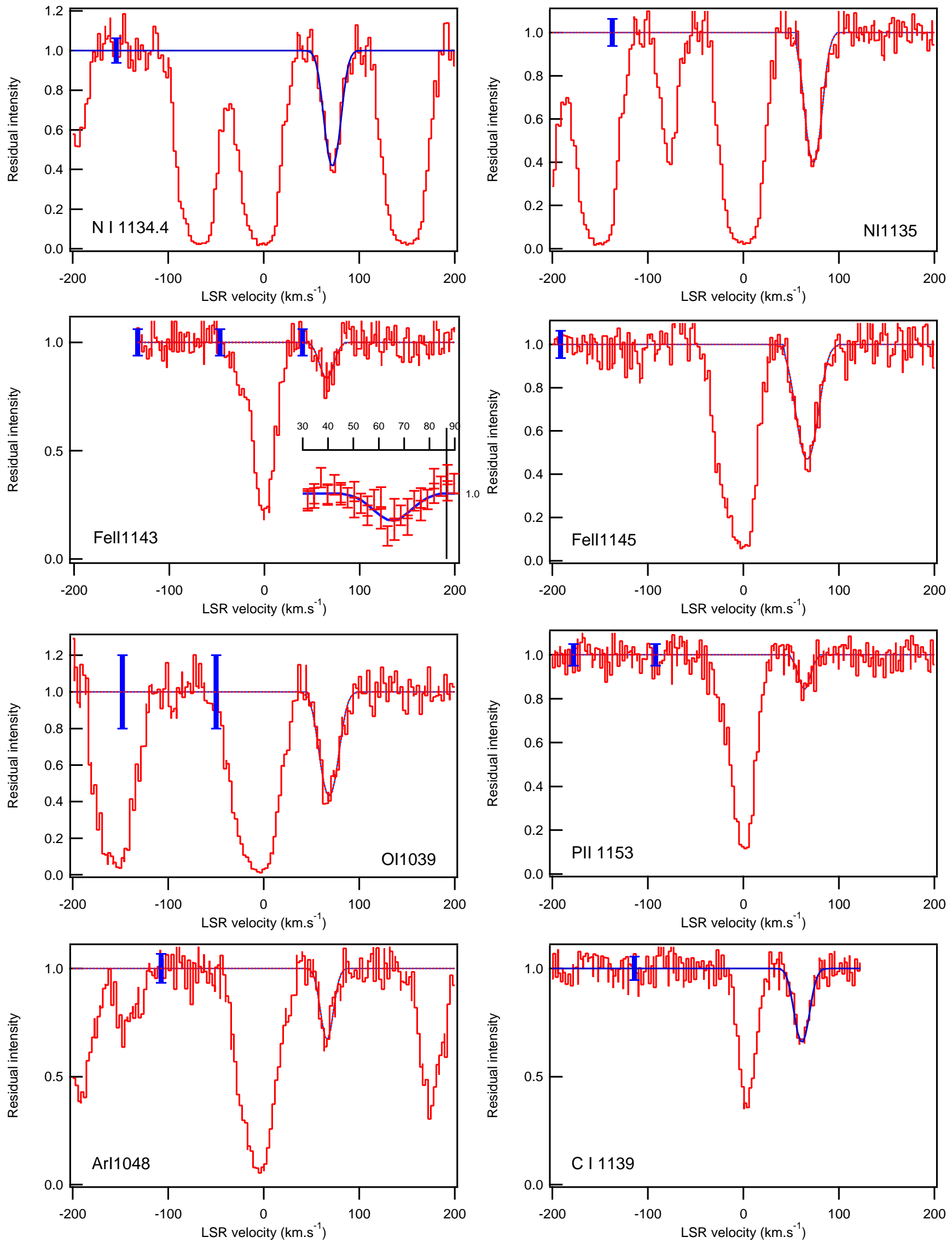

Fig. 3. FUSE spectra absorption profile fitting for HD 47240; results are given in residual intensity. Solid bars indicate typical error sizes to the continuum level fits. 
Table 1. FUSE spectra - HD 47240 absorption line measurements.

\begin{tabular}{|c|c|c|c|c|c|}
\hline Line & $\begin{array}{c}W_{\lambda} \\
(\mathrm{m} \AA)\end{array}$ & $\begin{array}{c}V_{\mathrm{LSR}} \\
\left(\mathrm{km} \mathrm{s}^{-1}\right)\end{array}$ & $b$ & $\begin{array}{c}N \\
\left(10^{12} \text { at cm }^{-2}\right)\end{array}$ & $S / N$ \\
\hline C I $1139 \ldots \ldots$ & 28.2 & $61.2(0.6)$ & $9.6(0.9)$ & $182(34)$ & 19 \\
\hline N I $1134.4 \ldots .$. & 43.2 & $71.8(0.4)$ & $9.4(0.6)$ & $175(31)$ & 16 \\
\hline N I $1135 \ldots \ldots \ldots$ & 49.4 & $73.1(0.4)$ & $10.4(0.6)$ & 137 (18) & 16 \\
\hline O I $1039 \ldots \ldots . .$. & 47.5 & $68.4(2.3)$ & $11.7(3.1)$ & $724(42)$ & 15 \\
\hline O VI $1032 \ldots .$. & 74.9 & $2.3(0.8)$ & $17.3(1.1)$ & $82(14)$ & 11 \\
\hline$(u)$ & $<9$ & $\mathrm{HV}$ & & $<7$ & \\
\hline P II $1153 \ldots \ldots$ & 8.8 & $63.9(1.1)$ & $7.9(1.7)$ & $4(1)$ & 20 \\
\hline Ar I $1048 \ldots .$. & 20.9 & $66.4(0.6)$ & $8.5(1)$ & $9(2)$ & 16 \\
\hline Fe II $1143 \ldots .$. & 10.9 & $65.4(1.4)$ & $9.5(2.1)$ & $55(12)$ & 17 \\
\hline Fe II $1145 \ldots .$. & 53.5 & $66.6(0.5)$ & $14(0.7)$ & $58(7)$ & 16 \\
\hline
\end{tabular}

Table 2. IUE spectra - HD 47240 absorption line measurements.

\begin{tabular}{|c|c|c|c|c|c|}
\hline Line & $\begin{array}{c}W_{\lambda} \\
(\mathrm{m \AA}) \\
\end{array}$ & $\begin{array}{c}\begin{array}{c}V_{\mathrm{LSR}} \\
\left(\mathrm{km} \mathrm{s}^{-1}\right)\end{array} \\
\end{array}$ & $\overline{\bar{b}}$ & $\begin{array}{c}N \\
\left(10^{12} \mathrm{at} \mathrm{cm}^{-2}\right) \\
\end{array}$ & 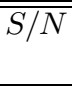 \\
\hline C II* $1336 \ldots \ldots$ & 70 & $59.2(0.9)$ & $12.1(1.8)$ & $171(25)$ & 28 \\
\hline C IV $1548^{(u)}$ & $<15$ & $\mathrm{HV}$ & & $<3$ & \\
\hline O I $1302 \ldots \ldots$ & 80 & $66.1(0.8)$ & $11.6(1.9)$ & $>350^{(s)}$ & 20 \\
\hline Mg II 3p 2796/2803 & $460 / 386$ & $57.7(1.5)$ & $11.9(2.7)$ & $>110^{(s)}$ & 17 \\
\hline Mg I $2852 \ldots \ldots \ldots$ & 107 & $66.7(2)$ & $5.7(6)$ & $1.6(5)$ & 14 \\
\hline Al II $1670 \ldots \ldots . .$. & 80 & $67.0(0.8)$ & $12.1(1.7)$ & $4.2(1)$ & 32 \\
\hline Al III $1855^{(u)} \ldots \ldots$ & $<12$ & $\mathrm{HV}$ & & $<50$ & \\
\hline Si II $1304 \ldots .$. & 99 & $66.1(1.2)$ & $13(2.4)$ & $150(14)$ & 18 \\
\hline Si IV $1394^{(u)} \ldots \ldots$. & $<24$ & $\mathrm{HV}$ & & $<3$ & 19 \\
\hline S II $1253 \ldots$. & 37 & $65.2(1.7)$ & $9.9(2.3)$ & $290(90)$ & 23 \\
\hline Fe II $1608 \ldots \ldots \ldots$ & 65 & $72(0.9)$ & $8.3(2.3)$ & $70(43)$ & 20 \\
\hline
\end{tabular}

(u)- Upper limits on the HV component. (s)- Saturated lines.
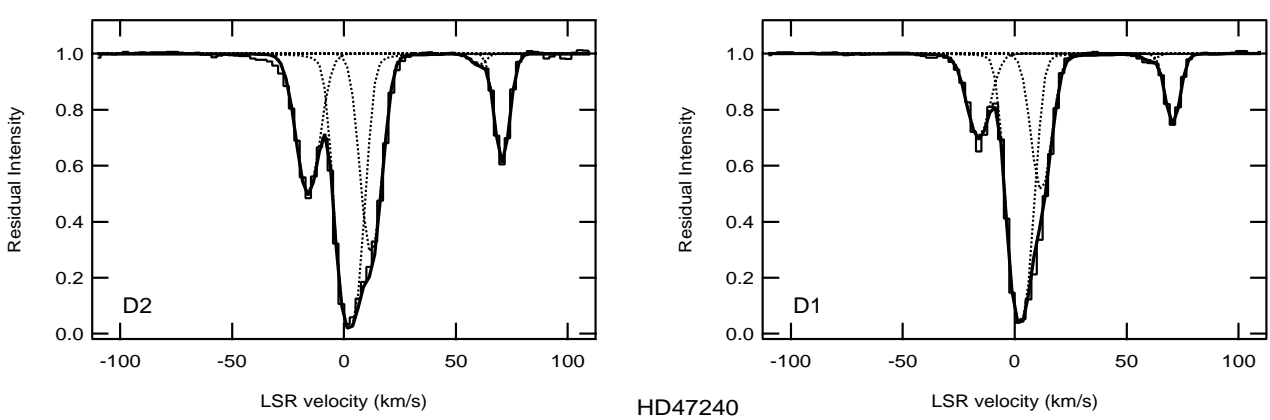

Fig. 4. Na I absorption profile fitting for HD 47240. Data taken from Sfeir (1999).

saturated. Unfortunately the HV components detected in both of the $\mathrm{Mg}$ II lines are saturated and in Table 2 we list a minimum value for $N(\mathrm{MgII})$ which will not be used in the subsequent analysis of element abundances in Sect. 5 due to its inherently large error estimate.

Our high resolution ground-based observations of the Ca II line towards HD 47240 indicate that this HV component is actually a blend of (at least) two absorbing clouds at $V_{\mathrm{LSR}} \sim+60$ and $V_{\mathrm{LSR}} \sim+71 \mathrm{~km} \mathrm{~s}^{-1}$. However, the lower resolution FUSE and IUE observations were unable to resolve these two clouds and it was found that the best fit to the HV component in all the UV data was obtained using a one-cloud absorption model centered at $V_{\mathrm{LSR}} \sim+65 \mathrm{~km} \mathrm{~s}^{-1}$.

\section{Discussion}

In this section we discuss the main physical and chemical aspects of the HV interstellar gas component observed in the far UV absorption line data, which is supplemented by both IUE and visible line data. 

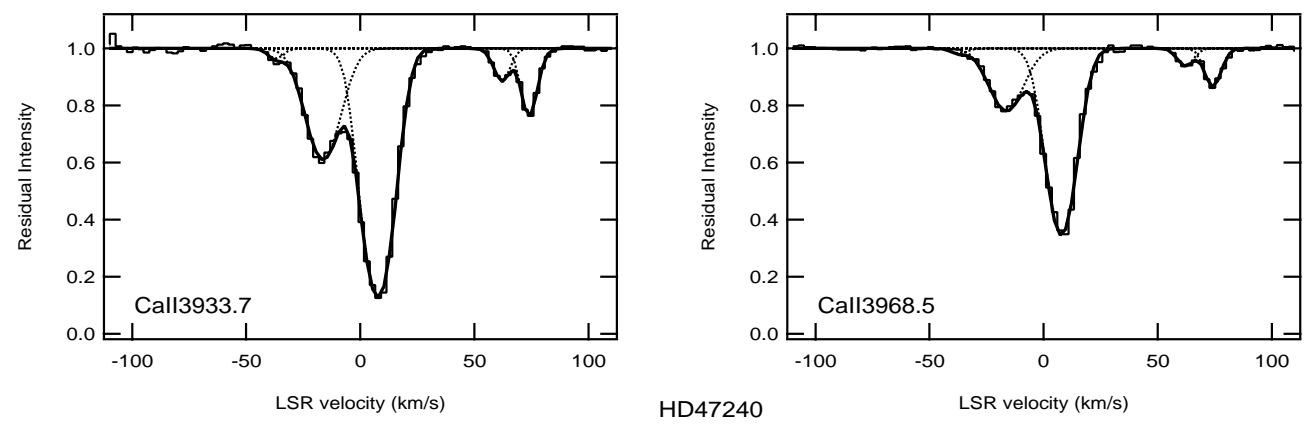

Fig. 5. Ca II absorption profile fitting for HD 47240. Data taken from Sfeir (1999).

Table 3. Na I (D2/D1) and Ca II doublet absorption line measurements for HD 47240.

\begin{tabular}{|c|c|c|c|c|c|c|}
\hline Line & $\begin{array}{c}W_{\lambda}(\mathrm{D} 2) \\
(\mathrm{m} \AA)\end{array}$ & $\begin{array}{c}W_{\lambda}(\mathrm{D} 1) \\
(\mathrm{m} \AA)\end{array}$ & $\bar{~} S / N$ & 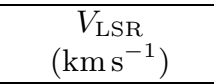 & $\bar{b}$ & $\begin{array}{c}N \\
\left(10^{11} \mathrm{at} \mathrm{cm}^{-2}\right)\end{array}$ \\
\hline Na I......... & 601.6 & 475.9 & 150 & $\begin{array}{c}-16(0.1) \\
2.4(0) \\
12.1(0.1) \\
59.6(1.4) \\
70.6(0) \\
\end{array}$ & $\begin{array}{l}5.6(0.1) \\
2.5(0.1) \\
4.4(0.1) \\
1.1(3.9) \\
1.9(0.1) \\
\end{array}$ & $\begin{array}{c}8.8(0.0) \\
3934(1256) \\
15.2(0.6) \\
0.5(6.6) \\
10.9(1.1) \\
\end{array}$ \\
\hline "Line & $\begin{array}{c}W_{\lambda}(\mathrm{K}) \\
(\mathrm{m} \AA) \\
\end{array}$ & $\begin{array}{c}W_{\lambda}(\mathrm{H}) \\
(\mathrm{m} \AA) \\
\end{array}$ & 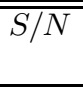 & $\begin{array}{c}V_{\mathrm{LSR}} \\
\left(\mathrm{kms} \mathrm{s}^{-1}\right) \\
\end{array}$ & $\bar{b}$ & $\begin{array}{c}N \\
\left(10^{11} \mathrm{at} \mathrm{cm}^{-2}\right) \\
\end{array}$ \\
\hline Ca II........ & 356.2 & 224.7 & 120 & $\begin{array}{c}-37.1^{(a)}(1.4) \\
-16.4(0.1) \\
7.6(0) \\
62(0.3) \\
74(0.1)\end{array}$ & $\begin{array}{c}1.6(2.4) \\
9.2(0.2) \\
7.3(0) \\
1.9(0.7) \\
2.1(0.3)\end{array}$ & $\begin{array}{c}0.5(1.3) \\
13.5(0.1) \\
50.1(0.1) \\
1.7(1.3) \\
8.3(2.8) \\
\end{array}$ \\
\hline
\end{tabular}

\subsection{The high velocity component}

Our FUSE observations have revealed a well-resolved high velocity absorption feature at $V_{\mathrm{LSR}} \sim+65 \mathrm{~km} \mathrm{~s}^{-1}$ in the O I, Ar I, N I, C I, Fe II and P II lines shown in the residual intensity profiles of Fig. 3. This HV feature is also well detected in the $I U E$ profiles of the nine near ultraviolet lines shown in Fig. 6 (O I, Al II, Si II, S II, Fe II, C II*, Mg I and Mg II) and in both the Na I and Ca II visible line profiles (Sfeir 1999). Note that we have been unable to detect a similar interstellar HV feature in the IUE spectra of the high ionization lines of Al III, C IV or Si IV. Upper limits for their equivalent width (derived from a conservative measurement of the local continuum noise) and their corresponding column density upper limit values can be found in Table 2 .

Inspection of the ionization potentials of the many lines exhibiting this HV absorption feature reveal that it is formed over a wide ionization range from $0<$ I.P. $<$ $23.3 \mathrm{eV}$ (defined by the Na I and S II line detections). Clearly all these ions cannot physically co-exist within the same HV cloud and thus, based on the velocity structure revealed by the high resolution $\mathrm{Na}$ I and Ca II observations, we conjecture that this HV feature may be composed of several ionized and neutral gas shells expanding at slightly different velocities away from the center of the SNR.
We note that the doppler $b$ values derived from the high resolution observations of both $\mathrm{Na}$ I and Ca II for the two resolved HV components are $<2.1 \mathrm{~km} \mathrm{~s}^{-1}$, indicating a gas temperature $<8000 \mathrm{~K}$. Although we have derived $b$ values in fitting the UV line data, their usefulness in determining reliable gas temperatures is limited due to the dominant contribution of the instrumental resolution of both IUE and FUSE spectrographs in fitting the blended components in these spectral lines. Hence, for the HV component observed with FUSE, the average $b$ value is found to be $\sim 10 \mathrm{~km} \mathrm{~s}^{-1}$, implying a typical gas temperature of $<90000 \mathrm{~K}$.

\subsection{O VI line}

In contrast to the low ionization interstellar lines, the high ionization absorption line profile of O VI shown in Fig. 7 is quite different in appearance. It consists of a single main absorption component centered at $V_{\mathrm{LSR}}=+2.7 \mathrm{~km} \mathrm{~s}^{-1}$ and (given the present signal-to-noise of the FUSE spectrum) is not accompanied by a high positive velocity component to a limit of $N(\mathrm{O} \mathrm{VI})<7 \times 10^{12} \mathrm{~cm}^{-2}$. We recall also that no HV components were detected for the high ionization lines of Si IV, C IV and Al III in our extracted IUE spectra. Thus, it would appear that since 

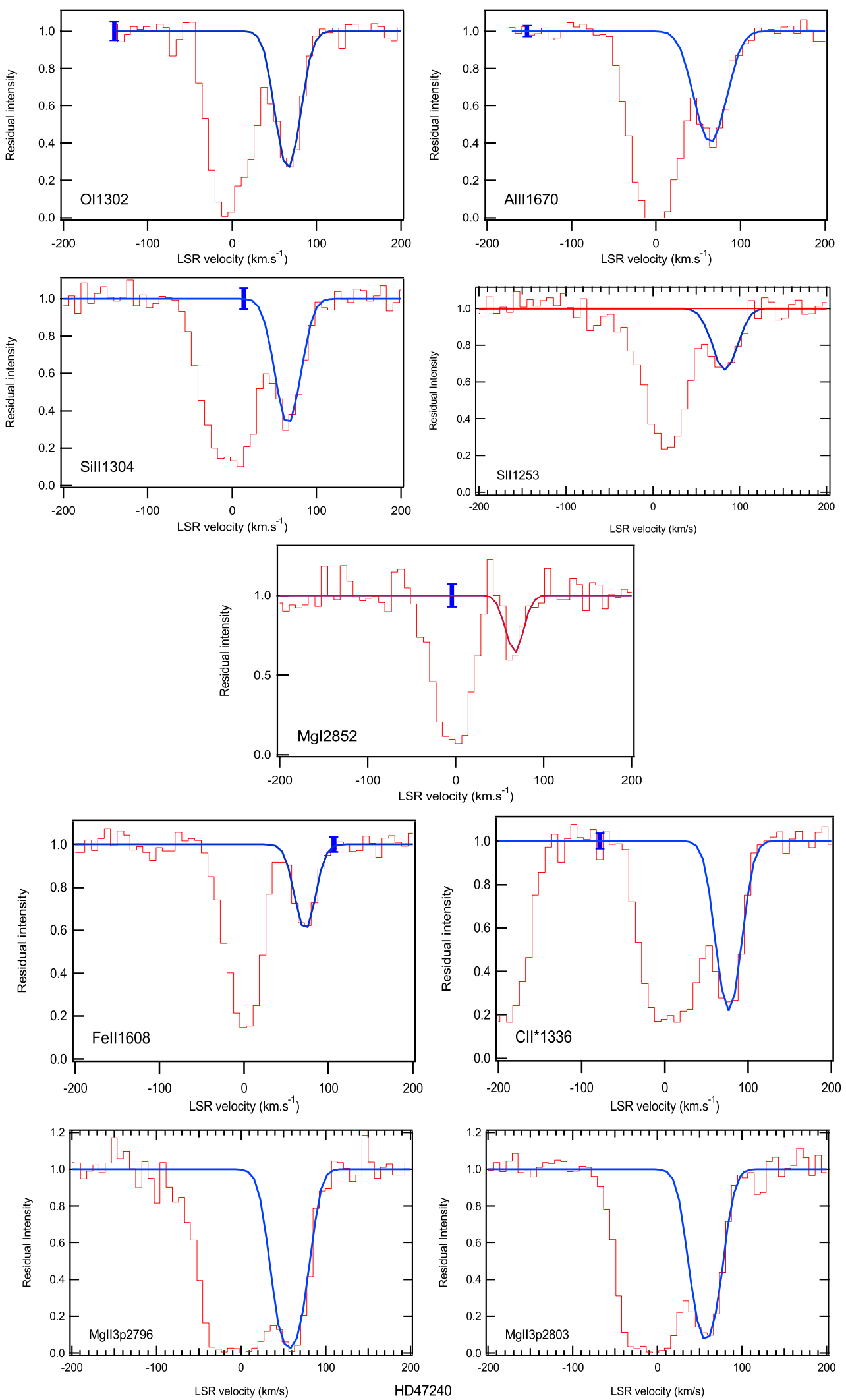

Fig. 6. IUE absorption profile fitting for HD 47240; results are given in residual intensity. Solid bars indicate typical error sizes to the continuum level fits. 


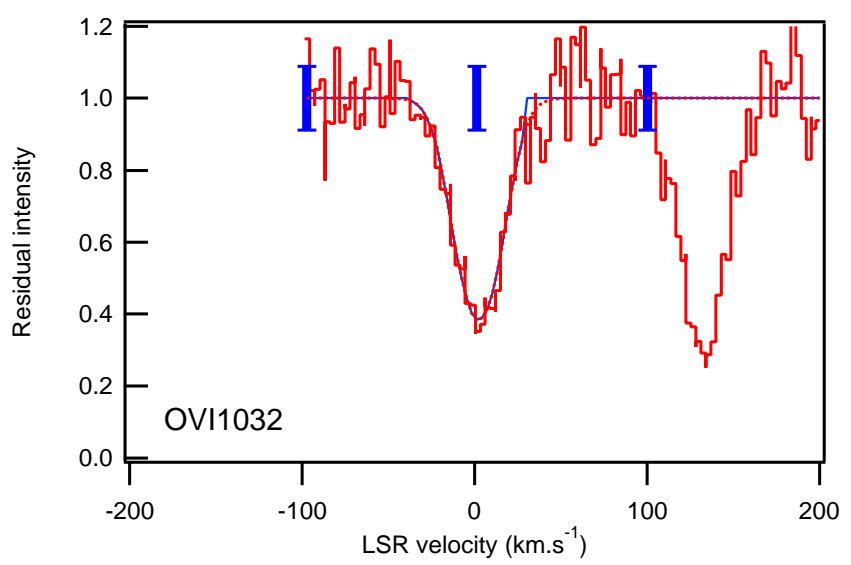

Fig. 7. FUSE spectra O VI absorption profile fitting for HD 47240; results are given in residual intensity. Solid bars on the continuum level indicate typical error size to the continuum level fit. The absorption line at $\sim 150 \mathrm{~km} \mathrm{~s}^{-1}$ is $\mathrm{H}_{2}$ $1032.356 \AA$ A.

the O VI absorption is formed over a restricted velocity range $\left(-30\right.$ to $\left.+30 \mathrm{~km} \mathrm{~s}^{-1}\right)$, it is most likely that it can be associated with the high ionization gas found in the intervening $1400 \mathrm{pc}$ of the general interstellar medium towards the Monoceros Loop and is not associated with the SNR itself. This lack of detectable high-ionization (hightemperature) HV absorbing SNR gas is somewhat surprising since SNR shock waves strongly affect the density and temperature structure of the ambient interstellar medium. Additionally, high-velocity features have been detected in high-ionization UV absorption lines towards other remnants such as the Vela SNR (Jenkins et al. 1976, 1984) and the Loops I and IV SNRs (Sembach \& Savage 1997), and also high-velocity O VI, C IV and Si IV features are all routinely observed in emission towards (bright filament) SNRs such as the Cygnus Loop (Long et al. 1992), the Vela SNR (Raymond et al. 1997) and Puppis A (Blair et al. 1995). However we note the absence of detections of such high-ionization features in the UV absorption spectra recorded towards the Shajn 147 SNR (Phillips \& Godhalekar 1983). This SNR has a (Sedov) age of $\sim 9 \times 10^{4}$ years which is similar to that of the Monoceros Loop SNR (Graham 1982), but is far older than both the Vela and Cygnus Loop SNRs. Similarly both Shajn 147 and the Monoceros Loop have HV components with velocities $<80 \mathrm{~km} \mathrm{~s}^{-1}$, such that this magnitude of shock velocity may be insufficient to produce detectable O VI. This is supported by the the calculations of Shelton (1998) which show that after $\sim 2.5 \times 10^{5}$ years a typical SNR shock is too weak to heat the interstellar gas to more than $10^{5} \mathrm{~K}$ and the high-stage ions are no longer found near the shock front. Instead, they lie at the edge of the hot SNR bubble and such gas is rapidly cooling and recombining through the $\mathrm{O}$ VI, N V and C IV ions.
Clearly, as more FUSE spectra of stars in the Monoceros Loop region become available to us, the spatial extent and range of ionization of the various absorbing components present throughout this evolved remnant will become clearer. For example, if the HV feature detected towards HD 47240 is ubiquitously detected in the FUSE absorption spectra of the other three target stars towards the Monoceros Loop, then this would argue strongly in favor of it being associated with a dense SNR shell arising from radiative cooling during the Sedov-Taylor phase. Such observations will be of particular importance in answering the outstanding question as to why there is (lowlevel) soft X-ray emission from the Monoceros Loop SNR but (as yet) no detectable O VI absorption/emission.

\section{Element abundances in the high velocity component}

Supernovae explosions can significantly alter the elemental abundances of the ambient interstellar gas through both the dissemination of the newly nucleosynthesized material and through the sputtering and evaporation of interstellar dust grains by the incident SNR shock front. WJ reported an anomalous $N(\mathrm{Na} \mathrm{I}) / N(\mathrm{Ca} \mathrm{II})$ ratio of $\sim 0.7$ for the $\mathrm{HV}$ absorption component towards HD 47240 which is typical for high velocity gas in the general ISM (Siluk \& Silk 1976). Since sodium depletion is thought to be approximately constant in the diffuse ISM (Jura 1976), then the low $\mathrm{Na}$ I/Ca II ratio can be attributed to the removal of adsorbed calcium atoms from dust grain surfaces by interstellar shock waves. However, our higher resolution visible observations reveal the $\mathrm{HV}$ component to be split into (at least) two components whose Na I/Ca II ratios are 0.29 $\left(V=+60 \mathrm{~km} \mathrm{~s}^{-1}\right)$ and $1.3\left(V=+70 \mathrm{~km} \mathrm{~s}^{-1}\right)$ respectively. This variation in Na I/Ca II column density ratio between the two cloud components can be easily seen in Figs. 4 and 5 , and suggests that the gas in the inner (slower) regions of the SNR may well have been more affected by the SNR blast wave.

Assuming that solar abundances reflect the present element abundances in the interstellar medium, then the depletion factor $(\delta)$ of a given element in the gas phase can be defined as $\delta_{\mathrm{X}}=\log (N(\mathrm{X}) / N(\mathrm{H}))_{\text {cloud }}-$ $\log (N(\mathrm{X}) / N(\mathrm{H}))_{\text {sun }}$. Element abundances may be thus determined if the column density of hydrogen, $N(\mathrm{H})$, is known for each cloud component along the line-of-sight to HD 47240. Unfortunately such values are, as yet, unavailable from Ly $\alpha$ or H I radio $21 \mathrm{~cm}$ studies. Thus, assuming that sulphur $(\mathrm{S})$ is undepleted in the interstellar gas (as is normally found for lines-of-sight with relatively low reddening), and that it has a solar abundance relative to hydrogen, then the (equivalent) hydrogen column densities for each absorption component may be determined. This also assumes that the S II lines (around $1255 \AA$ ) are the dominant ionization state of interstellar sulphur in line-of-sight towards the Monoceros Loop.

The HV component at $V=+65 \mathrm{~km} \mathrm{~s}^{-1}$ was clearly detected in the SII $(1253 \AA)$ line of the IUE spectrum of 
Table 4. Comparison of depletions for the line-of-sight to HD 47240.

\begin{tabular}{ccccc}
\hline \hline Element & $\begin{array}{c}\text { Mon Loop } \\
\text { HVC Depletion }\end{array}$ & $\begin{array}{c}\text { Vela SNR } \\
\text { HVC Depletion }\end{array}$ & $\begin{array}{c}\text { Cold ISM Gas } \\
\text { Depletion }^{(2)}\end{array}$ & $\begin{array}{c}\text { Warm ISM Gas } \\
\text { Depletion }^{(2)}\end{array}$ \\
\hline $\mathrm{S}$ & 0 & 0 & 0 & 0 \\
$\mathrm{Mg}$ & $\mathrm{N} / \mathrm{A}$ & $\mathrm{N} / \mathrm{A}$ & -1.24 to -1.56 & -0.73 to -0.9 \\
$\mathrm{Si}$ & $-0.57(0.17)$ & -0.5 & -1.31 & -0.35 to -0.51 \\
$\mathrm{Fe}$ & $-0.96(0.21)$ & -0.55 & -2.09 to -2.27 & -1.19 to -1.24 \\
$\mathrm{~N}$ & $-0.98(0.21)$ & -1.72 & -0.07 & -0.2 \\
$\mathrm{O}$ & $-1.21(0.16)$ & -1.14 & -0.39 & -0.4 \\
$\mathrm{P}$ & $-0.17(0.24)$ & -0.58 & -0.5 & -0.23 \\
$\mathrm{Al}$ & $-1.05(0.24)$ & -1.2 & -2.4 & -1.1 \\
$\mathrm{Ar}$ & $-0.77(0.24)$ & $\mathrm{N} / \mathrm{A}$ & -0.48 & $\mathrm{~N} / \mathrm{A}$ \\
\hline \multicolumn{5}{c}{$(1)-$ Jenkins et al. (1984), Jenkins et al. $(1998)}$. \\
\hline
\end{tabular}

HD 47240. It was also seen in the SII $1259 \AA$ line profile, but was contaminated by the nearby strong interstellar line of Si II $(1260 \AA)$. We failed to detect an HV component in the weaker SII line at $1251 \AA$ line. Using the derived column density, $\log N(\mathrm{~S})=14.46 \mathrm{~cm}^{-2}$, we calculate an equivalent value of $\log N(\mathrm{HI})=19.20 \mathrm{~cm}^{-2}$ for the $\mathrm{HV}$ component. Using this value of $N(\mathrm{H} \mathrm{I})$ we derive other element abundances under the assumption that the observed ionization stages for each element are the dominant ones in the ISM. The resultant element depletion patterns (relative to that of $\mathrm{S}$ ) for the $\mathrm{HV}$ component are shown in Table 4. For comparison purposes we also show the patterns of element depletion for the (younger) Vela SNR (Jenkins et al. 1984, 1998) and the depletion patterns associated with both warm and cool interstellar disk gas as listed by Savage \& Sembach (1996) and Welty et al. (1999).

In regions of the interstellar medium that have been highly disturbed one may expect that $\mathrm{O}, \mathrm{N}$ and $\mathrm{S}$ will be relatively undepleted, and that $\mathrm{Mg}, \mathrm{Si}$ and $\mathrm{Fe}$ will be depleted onto refractory grains that are gradually returned to the gas phase by sputtering and by grain-grain collisions. We note that for both the Monoceros Loop HV component and the Vela HV gas that the refractory elements of Fe, Si and $\mathrm{Al}$ are less depleted than that found for the cold ISM disk gas, suggesting that grain sputtering has infact returned these elements back to the ambient SNR interstellar gas. These levels of $\mathrm{Fe}, \mathrm{Si}$ and $\mathrm{Al}$ depletion in both of the Vela and Monoceros Loop SNR HV gas clouds are more consistent with that found in the warm disk gas of the interstellar medium, which is generally characterized by gas with temperatures of $\sim 8000 \mathrm{~K}$.

In contrast, we find that the elements of $\mathrm{N}, \mathrm{O}$ and Ar are more depleted in both the Vela and Monoceros Loop HV gas than that generally found for both the cold and warm ISM. Jenkins et al. (1998) have argued that the strong deficiencies of both $\mathrm{N}$ and $\mathrm{O}$ in $\mathrm{HV}$ gas components can probably be attributed to ionization effects in which the observed lines of N I, Ar I and O I are not the dominant states for these elements in the HV gas. However, we note from Bohigas (1983) that a gradual dilution of nitrogenrich SNR gas filaments is expected as a SNR ages and the supernova ejecta are enriched with swept-up elements from the surrounding ISM. Finally, we note the relatively small depletion of phosphorus in the Monoceros Loop HV gas which is similar to that found for the warm phase of the ISM disk gas. This pattern of near solar-system abundance for $\mathrm{P}$ (and $\mathrm{Zn}$ ) has also been observed towards the disk stars $\mu$ Columbae and $\zeta$ Oph (Howk et al. 1999).

\section{Energetics and remnant age}

For the case of the well-studied Vela SNR, the overall behavior of the velocities of the absorption components detected towards 45 stars was such that no ubiquitous component could be identified that was consistent with a radial expansion of the SNR (Jenkins et al 1984). The $\mathrm{HV}$ interstellar lines detected towards Vela were thought to be formed in shock-accelerated clouds, rather than behind a shock expanding into a uniform medium. However, for the Shajn 147 SNR (in which only two lines-of-sight have been sampled using the IUE satellite) a common $\mathrm{HV}$ component at $V=+80 \mathrm{~km} \mathrm{~s}^{-1}$ was detected towards both stars observed by Phillips \& Gondhalekar (1983). Thus, since we have (as yet) only sampled a single lineof-sight through the Monoceros Loop SNR it is premature (although tempting) to associate the HV feature at $+65 \mathrm{~km} \mathrm{~s}^{-1}$ seen towards HD 47240 with a global expansion of the outer SNR shell. Until absorption spectra along several more lines-of-sight are obtained towards the Monoceros Loop we cannot, as yet, distinguish whether this HV feature can be associated with shocks within dense clouds that have been overtaken by the much faster SNR shock wave, or whether the high velocity motion is due to a gas cloud that has been accelerated by the post-shock flow at some earlier epoch.

In addition, we note that although HV high ionization interstellar lines of CIV and SiIV were observed towards stars in the Vela SNR, such detections were limited to 
about $40 \%$ of the lines-of-sight sampled. No HV components were detected in the high ionization lines seen observed towards Shajn 147. Thus, in the case of SNRs the absence of presence does not always indicate the presence of absence for highly ionized HV components!

For a SN explosion energy of $3 \times 10^{50} \mathrm{erg}$ occuring in a uniform medium of ambient density, $1 \mathrm{~cm}^{-3}$, the relationship between the SNR shell velocity $(V)$ and that the remnant age, $t$ (in units of $10^{5}$ years) is given by Chevalier (1974) as:

$V=66.5 \times t^{-0.69} \mathrm{~km} \mathrm{~s}^{-1}$.

Under the simple (and as yet unsupported) assumption that the $\mathrm{HV}$ component we have detected towards HD 47240 is representative of a SNR shell with a resultant nebular expansion velocity of $50 \mathrm{~km} \mathrm{~s}^{-1}$ (Davies et al. 1978), then we obtain an age for the Monoceros Loop SNR of $t=1.5 \times 10^{5}$ years, in agreement with previous estimates for this remnant by Graham et al. (1982). However, two important caveats must be taken into account in this age determination. The first is that the ambient interstellar density that the SNR is expanding into is unknown, and is probably inhomogenous by large magnitudes. Secondly, we note that Leahy et al. (1986) have derived a far younger age of $3 \times 10^{4}$ years for the Monoceros Loop SNR. This younger age was derived by matching a model with the shock radius, density $\left(\rho=0.001\right.$ atom $\left.\mathrm{cm}^{-3}\right)$ and temperature of the X-ray emitting gas. Thus, although at present our FUSE observations cannot provide a definite age for the Monoceros Loop remnant it would appear that an age of 30000 to 150000 years seems appropriate. This age range could be consistent with the intepretation that the $\mathrm{HV}$ feature we have observed in both the FUSE and IUE data represents that of an evolved shocked SNR shell with no associated high-ionization (high-temperature) absorption. As stated previously, such high-ionization features have thus far only been detected in the ultraviolet towards SNRs with an age $<15000$ years (Cygnus Loop and Vela SNR).

\section{Summary}

We have obtained a far ultraviolet spectrum of the star HD 47240 covering the wavelength range 990-1187 $\AA$ using the NASA FUSE satellite. Using these new FUV data supplemented by visible and IUE archival absorption spectra taken towards this star, we have detected a high-velocity absorption component at $V_{\mathrm{LSR}}=+65 \mathrm{~km} \mathrm{~s}^{-1}$ whose origin can be associated with the highly disturbed SNR gas. This high-velocity feature is formed only in lines with an ionization potential range up to $23.3 \mathrm{eV}$. High spectral resolution visible observations of this feature reveal a more complex velocity structure (with at least two velocity components at $V_{\mathrm{LSR}}=+60$ and $+71 \mathrm{~km} \mathrm{~s}^{-1}$ ), suggesting that it is composed of several ionized and neutral gas shells expanding at slightly different velocities.

This high-velocity feature has not been detected in any of the high-ionization absorption lines of O VI, C IV or Si IV. Similar non-detections of these high ionization lines have been reported for HV gas associated with the Shajn 147 SNR (which is of a similar age to the Monoceros Loop), whereas both detections and non-detections of $\mathrm{HV}$ high ions have been reported towards the (younger) Vela SNR which is expanding at velocities $>100 \mathrm{~km} \mathrm{~s}^{-1}$. Thus, until more lines-of-sight are sampled towards the Monoceros Loop SNR this present lack of HV high ion line detectability cannot be attributed solely to the low $\left(V<80 \mathrm{~km} \mathrm{~s}^{-1}\right)$ shock velocity and evolved nature of the expanding remnant gas.

We have derived elemental abundances (relative to that of S) for gas associated with the HV component. A pattern in which the refractory elements of Fe, Si and $\mathrm{Al}$ are all less depleted (i.e. more abundant) than in the cold, interstellar disk gas has been found. This pattern of overabundance of refractory elements found for both the Monoceros Loop and Vela SNRs is consistent with the levels of depletion found in warm disk gas, and is probably due to grain sputtering processes. In contrast $\mathrm{N}, \mathrm{O}$, and Ar are all more depleted in the Vela and Monoceros Loop SNR HV gas than in both the cold and warm interstellar disk gas. This apparent element deficiency can be attributed to ionization effects of the SNR gas. The element phosphorus is essentially undepleted in the highvelocity gas, in agreement with observations of interstellar gas in the galactic disk.

Finally, we believe that an age of 30000 to 150000 years for the Monoceros Loop SNR is appropriate. Such an evolved remnant appears to possess absorption properties more like those of the Shajn 147 SNR as opposed to those of the far younger (age $<15000$ years) Cygnus and Vela SNRs.

Acknowledgements. We wish to thank the NASA FUSE Operations and Science Center at the Johns Hopkins University which is operated for NASA under contract NAS532985. Particular thanks go to Dr. Bill Blair, Dr. Robin Shelton and Dr. Ed Jenkins for very useful discussions and important suggestions that have greatly improved this paper. DS, SS and BYW all acknowledge funding through the NASA FUSE science support contract with the Experimental Astrophysics Group at the Space Sciences Laboratory, UC Berkeley.

\section{References}

Blair, W., Raymond, J., Long, K., \& Kriss, G. 1995, ApJ, 454, 35

Bohigas, J. 1983, Rev. Mex. Astr. Ap., 5, 271

Chevalier, R. 1974, ApJ, 188, 501

Cowie, L. L., McKee, C., \& Ostriker, J. 1981, ApJ, 247, 908

Danks, A., \& Sembach, K. 1995, AJ, 109, 2627

Davies, R., Elliott, K., Goudis, C., Meaburn, J., \& Tebbutt, N. 1978, A\&AS, 31, 271

Fesen, R., Blair, W., \& Kirschner, R. 1985, ApJ, 292, 29

Graham, D. A., Haslam, C., Salter, C., \& Wilson, W. 1982, A\&A, 109, 145

Howk, J. C., Savage, B. D., \& Fabian, D. 1999, ApJ, 525, 253 
Jaffe, T. R., Bhattacharya, D., Dixon, D., \& Zych, A. 1997, ApJ, 484, L129

Jenkins, E. B., Wallerstein, G., \& Silk, J. 1976, ApJS, 32, 681 Jenkins, E. B., Wallerstein, G., \& Silk, J. 1984, ApJ, 278, 649 Jenkins, E. B., et al. 1998, ApJ, 492, L147

Jura, M. 1976, ApJ, 206, 691

Kuchar, T. A., \& Bania, T. M. 1993, ApJ, 414, 664

Leahy, D. A., Naranan, S., \& Singh, K. 1986, MNRAS, 220, 501

Long, K. S., et al. 1992, ApJ, 400, 214

Moos, H. W., et al. 2000, ApJ, 538, L1

Morton, D. C. 2001, in preparation

Odegard, N. 1986, ApJ, 301, 813

Phillips, A. P., \& Gonhalekar, P. 1983, MNRAS, 202, 483
Raymond, J. C., et al. 1997, ApJ, 482, 881

Rodriguez-Pascual, P., Gonzalez-Riestra, R., Schartel, N., \& Wamstecker, W. 1999, A\&AS, 139, 183

Sahnow, D. J., et al. 2000, ApJ, 538, L7

Savage, B. D., \& Sembach, K. 1996, ARA\&A, 34, 279

Sembach, K., \& Savage, B. 1997, ApJ, 480, 216

Sfeir, D. M. 1999, Ph.D. Thesis, University of Paris 6

Sfeir, D. M., Lallement, R., Crifo, F., \& Welsh, B. Y. 1999, A\&A, 346, 785

Siluk, R., \& Silk, J. 1974, ApJ, 192, 51

Shelton, R. L., ApJ, 504, 785

Wallerstein, G., \& Jacobsen, T. S. 1976, ApJ, 207, 53

Welty, D., Frisch, P. C., Sonneborn, G., \& York, D., ApJ, 512, 636 